\title{
Dietas artificiais incorporadas ou não a colmos triturados de variedades de cana-de-açúcar na biologia de Diatraea saccharalis (F.) (Lepidoptera: Crambidae)
}

\section{Addition of shredded stem of sugar cane varieties to artificial diets on the biology of Diatraea saccharalis (F.) (Lepidoptera: Crambidae)}

\author{
Arlindo Leal Boiça Junior’; Aluízio Fumagalli Leonelo²; \\ Flávio Gonçalves de Jesus ${ }^{3 *}$
}

\begin{abstract}
Resumo
O efeito de dietas artificiais incorporadas ou não com colmos triturados de variedades de cana-deaçúcar na biologia de Diatraea saccharalis (Fabr., 1794) (Lepidoptera, Crambidae) foi estudado. O experimento foi conduzido no laboratório de Resistência de Plantas a Insetos do Departamento de Fitossanidade da FCAV-UNESP, Campus de Jaboticabal, SP, no ano de 2007, utilizando-se dietas com diferentes concentrações de seus componentes $(100,75,50$ e $25 \%$ da concentração dos componentes de uma dieta padrão). $\mathrm{O}$ experimento foi conduzido em delineamento inteiramente casualizados em esquema fatorial 4 X 3 , sendo 4 concentrações de dietas e 3 tratamentos onde continham ou não partes de colmo, correspondendo a 12 tratamentos e 6 repetições. Foram avaliados o período e viabilidade larval, pupal e total; peso larval com 15 dias de idade; peso de pupas com 24 horas de idade e longevidade de adultos sem alimentação. A dieta com $100 \%$ da concentração de seus componentes é mais favorável ao desenvolvimento de $D$. saccharalis, enquanto aquela com $25 \%$ é menos favorável. A dieta com $100 \%$ da concentração de seus componentes associada a presença de colmo da variedade SP80-3280 (suscetível) proporciona maior peso larval. Os pesos larval e pupal do inseto são afetados negativamente quando incorpora-se na dieta colmos secos triturados da variedade RB83-5486 (resistente). A dieta com $50 \%$ da concentração de seus componentes proporciona, quanto ao peso larval, melhor discriminação entre os genótipos resistente e suscetível à broca da cana-de-açúcar.
\end{abstract}

Palavras-chave: Insecta, broca da cana-de-açúcar, resistência de plantas

\begin{abstract}
It was studied the incorporation or not of shredded stems of sugarcane varieties to artificial diets and their effect in the biology of Diatraea saccharalis (Fabr., 1794) (Lepidoptera: Crambidae) under laboratory conditions. The experiment was carried out at the Department of Plant Protection of FCAVUNESP, Jaboticabal, São Paulo, Brazil, in 2007, using diets with different amounts of their components (100, 75,25 e $25 \%$ of concentration of the standard diet). The experiment had a complete randomized design in a $4 \times 3$ factorial scheme, using 4 concentrations of diets and three treatments, in a total of 12 , with 6 replications. The larval, pupal an total periods and viability were evaluated, as well as the larval weight at 15 days, the pupal weight after 24 hours and the longevity of adults without feeding. The diet
\end{abstract}

1 Prof. da Faculdade de Ciências Agrárias e Veterinárias, UNESP, Departamento de Fitossanidade. Jaboticabal, SP. E-mail: aboicajr@fcav.unesp.br

2 Aluno do Curso de Graduação, Faculdade de Ciências Agrárias e Veterinárias, UNESP, Departamento de Fitossanidade. Jaboticabal, SP. E-mail:1 eonelo@yahoo.com.br

3 Prof. do Instituto Federal Goiano, Campus Urutaí, Rodovia Prof. Geraldo Silva Nascimento Km 2,5, Cep: 75790-000, UrutaíGo, E-mail: fgjagronomia@zipmail.com.br

* Autor para correspondência

Recebido para publicação 18/09/09 Aprovado em 03/01/11 
with $25 \%$ concentration of the components compared to the standard diet had a negative effect in the development of $D$. saccharlis larvae. Shredded dry stalks incorporated or not to the diet affected only the larval and pupal weight whereas the larval weight was more affected when the resistant (RB835486) and susceptible (SP80-3280) varieties due to the use of diet with 50\% of its components. The diet with $100 \%$ concentration of its components are more favorable to the development of $D$. saccharalis, with $25 \%$ while that is less favorable. The diet with $100 \%$ concentration of the components associated with the presence of SP80-3280 (susceptible) stem variety provides greater larval weight. The weights of larvae and pupae of insects are negatively affected when RB83-5486 (resistant) shredded dry stem variety is incorporated into the diet. regarding to larval weight the diet with $50 \%$ of the concentration of its components provides better discrimination between genotypes resistant and susceptible to sugarcane borer.

Key words: Insecta, sugarcane borer, host plant resistance

\section{Introdução}

A cultura da cana-de-açúcar (Saccharum officinarum L.), devido à grande importância que assumiu nos últimos anos por conseqüência da alta produção alcançada, disseminou-se por todos os estados brasileiros, tendo sido conduzida nas mais diferentes condições climáticas, solo, técnicas e tratos culturais. Trata-se de uma cultura de grande importância econômica e social para o Brasil e, em particular, para o Estado de São Paulo, cuja área cultivada é de aproximadamente 3,96 milhões de hectares, com $62 \%$ de todo o açúcar e $60 \%$ de álcool produzido no país (AGRIANUAL, 2009).

Segundo Boiça Junior, Lara e Bellodi (1997), apesar da rusticidade que a cultura apresenta, defronta-se com uma série de problemas agronômicos e dentre estes, pode-se citar a incidência de pragas e, em particular, a presença acentuada da broca Diatraea saccharalis (Fabr., 1794) (Lepidoptera, Crambidae), considerada praga chave da cultura.

Gallo et al. (2002) afirmam que as lagartas causam prejuízos diretos pela abertura de galerias que ocasionam perda de peso na cana e morte das gemas, causando falhas na germinação. Quando a broca faz galerias transversais seccionando o colmo, elas provocam o tombamento da cana pelo vento e nas canas jovens o ataque da broca provoca o secamento dos ponteiros, conhecido por "coração morto".
Ainda se tratando de danos, existem aqueles tidos como indiretos, uma vez que através dos orifícios e galerias provocados pela praga, ocorre a penetração de fungos que causam a podridão vermelha do colmo, na qual pode abranger toda a região compreendida entre as diversas galerias. Esses fungos causadores da podridão vermelha são Colletotrichum falcatum Went e Fusarium moniliforme Sheldon, e provocam a inversão da sacarose, diminuição da pureza do caldo e provoca contaminações no processo de fermentação alcoólica, dando menor rendimento em açúcar e álcool.

Devido à arquitetura das plantas, espaçamento e hábito de ataque do inseto, torna-se muito difícil a utilização de inseticidas no controle da praga, além do que pode modificar em muito o ecossistema vigente na área de cultivo (TERÁN; PRECETTI; DERNEIKA, 1983).

Boiça Junior, Lara e Bellodi (1997), citam que o uso de variedades resistentes apresenta inúmeras vantagens, entre elas as de que a planta resistente é geralmente compatível com a aplicação de outras táticas de manejo do inseto, incluindo o controle biológico e químico, além de ser considerado como o método ideal de controle, uma vez que não altera o ecossistema, não necessita do conhecimento específico por parte do agricultor, não onera os custos de produção e mantém a praga abaixo dos níveis de dano econômico (LARA, 1991). 
O emprego de variedades resistentes à $D$. saccharalis, é a prática mais econômica para o seu controle, sem causar nenhum desequilíbrio ao ecossistema (LARA, 1991), tendo ainda, importância equivalente ao controle químico, em termos de controle das populações da broca da cana (BESSIN et al., 1990).

No Brasil as primeiras informações sobre diferentes graus de infestação da broca em variedade cultivadas na região de Piracicaba, foram fornecidas por Gallo (1954) citado por Gallo et al. (2002), destacando-se as maiores infestações para a CP17139 e CO419.

Derneika (1985) observou diferenças no comportamento de $D$. saccharalis alimentadas com diferentes variedades de cana-de-açúcar, sendo que as variedades SP71-345, SP71-6113 e SP711081 revelaram-se moderadamente resistentes, enquanto que as CP51-22, SP71-3146 e SP71-5574 apresentaram-se altamente suscetíveis ao ataque da broca.

Bellodi (1993) testou dietas artificiais à base de feijão incorporadas com partes de colmos de canade-açúcar da variedade SP71-1081 (resistente) e da variedade SP71-3146 (suscetível). Nos parâmetros estudados foram observadas diferenças estatísticas para a duração e peso larval e duração da fase pupal, constatando-se maior duração dessas fases (27,9 e 11,1 dias, respectivamente) e menor peso de lagartas $(24,5 \mathrm{mg})$ em dietas contendo colmo da variedade resistente SP-1081, e ao contrário disso, nos indivíduos criados em dieta contendo colmo da variedade SP71-3146, a fase larval e pupal foram mais rápidas (26,8 e 9,2 dias, respectivamente) e as lagartas mais pesadas (30,4 mg), evidenciando um favorecimento no desenvolvimento da broca no material suscetível.

O presente trabalho teve por objetivo comparar dietas em concentrações diferentes de seus componentes, incorporadas ou não com partes de colmo de variedades resistente e suscetível e influência na biologia de D. saccharalis.

\section{Material e Métodos}

Local e instalação do experimento. O trabalho foi desenvolvido no Laboratório de Resistência de Plantas a Insetos do Departamento de Fitossanidade da Faculdade de Ciências Agrárias e Veterinárias UNESP, Campus de Jaboticabal, SP, realizando-se o experimento no período de agosto a dezembro do ano de 2007. Foram coletadas partes de colmos de plantas de duas variedades de cana-de-açúcar, cedidos pela Usina Alta Mogiana S/A Açúcar e Álcool, município de São Joaquim da Barra/SP. As variedades de cana-de-açúcar foram a RB83-5486 (CTC), resistente a D. saccharalis e SP80-3280 (COPERSUCAR), suscetível (BOIÇA JUNIOR; LARA; BELLODI, 1997). Foram retiradas partes dos colmos, os quais foram picados, secos em estufa a temperatura constante de $60^{\circ} \mathrm{C}$ por 48 horas, e posteriormente triturados. A casca também foi utilizada com o objetivo de evitar erro uma vez que a resistência da variedade RB83-5486 pode estar associada a componentes físicos e/ou químicos presentes.

As diferentes fases da biologia do inseto foram conduzidas sob condições controladas com temperatura de $25 \pm 1^{\circ} \mathrm{C}$; UR de $70 \pm 10 \%$; e fotofase de 12 horas, sendo a criação iniciada a partir de posturas de $D$. saccharalis fornecidas pelo Laboratório de Controle Biológico da Usina Santa Adélia S/A, município de Jaboticabal/SP.

Elaboração de dietas. Para a condução do experimento foram elaboradas dietas artificiais a base de feijão, normalmente empregada nos laboratórios (MARCONATO, 1988) com diferentes concentrações de seus componentes, sendo uma delas considerada dieta padrão, na qual colocouse $100 \%$ da concentração dos ingredientes, enquanto que nas demais adotou-se concentrações inferiores, com 75,50 e $25 \%$ da concentração dos componentes da dieta padrão, sendo que em todas foram incorporadas ou não colmo seco triturado de cada variedade de cana-de-açúcar. Os componentes com suas devidas quantidades da dieta padrão foram 
elaborados de acordo com a de Hesley e Hammond (1968).

No preparo do meio artificial padrão (100\%), primeiramente cozinhou-se $150 \mathrm{~g}$ de feijão em 800 $\mathrm{mL}$ de água. Após esta etapa, misturaram-se os outros componentes da dieta, exceto o ágar, agitando-se a mistura em liquidificador por aproximadamente 10 minutos. Concomitantemente, $400 \mathrm{~mL}$ de água destilada efervescente e o ágar foram colocados em um recipiente comum e agitados até uma perfeita homogeneização. A seguir foi adicionado aos ingredientes contidos no liquidificador e a mistura agitada por mais 3 minutos. Todo esse procedimento foi repetido para a elaboração das outras dietas, reduzindo apenas a concentração dos componentes, com exceção do ágar.

A seguir, parte de cada dieta foi distribuída em tubos de ensaio ( $8 \mathrm{~cm}$ de comprimento x $2,5 \mathrm{~cm}$ de diâmetro) e colocados em suportes. Após o resfriamento foram fechados com tampões de algodão e mantidos sob condições controladas (Temperatura: $25 \pm 1^{\circ} \mathrm{C}$; UR: $70 \pm 10 \%$; fotofase: 12 horas) por 24 horas estando prontos para receberem as lagartas recém-eclodidas.

A parte restante do meio artificial foi armazenada em bandejas de plástico (50 $\mathrm{cm}$ de comprimento $\mathrm{x}$ $30 \mathrm{~cm}$ de largura $\times 8 \mathrm{~cm}$ de altura) e mantidos em geladeira $\left(8^{\circ} \mathrm{C}\right)$ para posteriormente serem cortadas em pequenos cubos $(2 \mathrm{~cm}$ de comprimento $\mathrm{x} 2 \mathrm{~cm}$ de largura x $2 \mathrm{~cm}$ de altura), e serem utilizados na alimentação das lagartas nas placas de Petri, simulando o colmo da cana-de-açúcar no campo.

Biologia de D. saccharalis. As posturas vieram acondicionadas sobre papel filtro previamente umedecido e envolto em papel alumínio, para a proteção no transporte dos mesmos, e após a chegada foi mantidas em laboratório a temperatura de $25 \pm 1^{\circ} \mathrm{C}$; UR de $70 \pm 10 \%$; e fotofase de 12 horas, até a eclosão das lagartas. Após a eclosão, com o auxílio de um pincel umedecido em água destilada, foram introduzidas 5 lagartas no interior de tubos de ensaio ( $8 \mathrm{~cm}$ de comprimento x 2,5 cm de diâmetro) contendo cada um sua respectiva dieta artificial. Em seguida os tubos de criação foram tampados com chumaços de algodão hidrófilo, resultando em 12 tratamentos (4 concentrações de dieta e 3 com ou sem partes de colmo de variedades) e 6 repetições com 5 lagartas cada, totalizando portanto 360 lagartas de D. saccharalis.

As lagartas permaneceram nos tubos de ensaio por 15 dias, sendo em seguida transferidas para placas de Petri $(1,5 \mathrm{~cm}$ de altura $\mathrm{x} 12,5 \mathrm{~cm}$ de diâmetro) onde continuaram recebendo as mesmas dietas na forma de pequenos cubos até a pupação.

Ao se transformarem em pupas, foram transferidas para tubos de PVC $(10 \mathrm{~cm}$ de altura x $10 \mathrm{~cm}$ de diâmetro), cobertos na parte superior por tecido "voil", permitindo a visão e a circulação do ar, e na parte inferior foi colocado papel filtro levemente umedecido para não ressecar o casulo, os quais deram origem aos adultos.

Aspectos biológicos estudados. Foram realizadas avaliações diárias a fim de se determinar a duração e a viabilidade do período larval, pupal e total; peso larval e pupal; e longevidade do adulto. Para a obtenção do peso larval, as lagartas foram pesadas aos 15 dias de idade enquanto que as pupas foram pesadas 24 horas após a formação do casulo. A longevidade dos adultos de D. saccharalis, mantidos sem alimentação, foi observada pelo número de dias de vida.

Análises estatísticas. $\mathrm{O}$ experimento foi conduzido em delineamento inteiramente casualizados em esquema fatorial 4 x 3, com 12 tratamentos e 6 repetições. Os dados obtidos foram submetidos à análise de variância pelo Teste de F. e quando significativas às médias foram comparadas pelo Teste de Tukey, ao nível de 5\% de probabilidade. Para a análise os dados da viabilidade larval e pupal e da viabilidade total foram transformados em arc $\operatorname{sen} \sqrt{X / 100}$. 


\section{Resultados e Discussão}

A duração dos períodos larval, pupal e total, foram afetadas pelas diferentes concentrações dos componentes nas dietas. Porém, quanto à presença ou não de partes de colmo de plantas das variedades de cana-de-açúcar, não foram constatadas diferenças entre esses tratamentos (Tabela 1).

Em relação a fase larval (Tabela 1), nota-se que as dietas com 100, 75 e $50 \%$ da concentração dos componentes proporcionaram diminuição significativa nos valores médios da duração desta fase sendo de 28,8;28,7 e 27,1 dias respectivamente, quando comparadas com o meio artificial com $25 \%$ da concentração dos componentes da dieta padrão, que apresentaram uma duração de 40,3 dias. Quanto à presença ou não de partes de colmos de variedades de cana-de-açúcar os valores médios de 31,4; 31,2 e 31,1 dias para as dietas incorporadas com a variedade SP80-3280, RB83-5486 e sem incorporação de colmos de variedades respectivamente, não diferiram entre si. Os resultados obtidos quanto a presença ou não de colmos de variedades de canade-açúcar, apresentaram valores superiores àqueles relatados por Terán, Sánchez e Precetti (1986), porém não diferiram entre si como àqueles.

Tabela 1. Valor médio do período larval, pupal e total (larval + pupal) de D. saccharalis, obtidos em quatro dietas de diferentes concentrações de seus componentes, incorporados ou não com partes de colmo de variedades de cana-deaçúcar. Temperatura: $25 \pm 1^{\circ} \mathrm{C}$; UR: $70 \pm 10 \%$; Fotofase: 12 horas.

\begin{tabular}{|c|c|c|c|}
\hline Concentração da dieta (D) & $\begin{array}{c}\text { Período larval } \\
\text { (dias) }\end{array}$ & $\begin{array}{c}\text { Período pupal } \\
\text { (dias) }\end{array}$ & $\begin{array}{c}\text { Período total } \\
\text { (dias) }\end{array}$ \\
\hline $100 \%$ & $28,8 \mathrm{~b}$ & $8,0 \mathrm{~b}$ & $35,2 \mathrm{~b}$ \\
\hline $75 \%$ & $28,7 \mathrm{~b}$ & $8,9 \mathrm{ab}$ & $37,2 \mathrm{~b}$ \\
\hline $50 \%$ & $27,1 \mathrm{~b}$ & $9,1 \mathrm{ab}$ & $35,9 \mathrm{~b}$ \\
\hline $25 \%$ & $40,3 \mathrm{a}$ & $9,2 \mathrm{a}$ & $47,9 \mathrm{a}$ \\
\hline $\mathrm{F}(\mathrm{D})$ & $107,7^{* *}$ & $3,3^{*}$ & $22,5^{* *}$ \\
\hline D.M.S & 2,2 & 1,2 & 4,7 \\
\hline \multicolumn{4}{|l|}{ Colmo (C) } \\
\hline Com variedade (SP80-3280) & $31,4 \mathrm{a}$ & $8,7 \mathrm{a}$ & . $38,3 \mathrm{a}$ \\
\hline Com variedade (RB83-5486) & $31,2 \mathrm{a}$ & $8,7 \mathrm{a}$ & . $39,5 \mathrm{a}$ \\
\hline Sem variedade & $31,1 \mathrm{a}$ & $9,0 \mathrm{a}$ & . $39,3 \mathrm{a}$ \\
\hline $\bar{F}(\mathrm{D})$ & . $0,1^{\mathrm{NS}}$ & $0,5^{\mathrm{NS}}$ & $0,3^{\mathrm{NS}}$ \\
\hline D.M.S & 1,7 & $.0,9$ & 3,7 \\
\hline $\mathrm{F}(\mathrm{D} \times \mathrm{C})$ & $2,2^{\mathrm{NS}}$ & .. $0,5^{\mathrm{NS}}$ & $0,3^{\mathrm{NS}}$ \\
\hline Desvio padrão & 2,5 & .. 1,3 & 5,3 \\
\hline $\mathrm{CV}(\%)$ & 8,0 & .. 15,0 & 13,6 \\
\hline
\end{tabular}

${ }^{1}$ Médias seguidas de mesma letra na coluna, para cada fator, não diferem entre si ao nível de 5\% de probabilidade pelo teste de Tukey. ${ }^{\mathrm{NS}}$ não significativo, ${ }^{* *}$ significativo a $1 \% \mathrm{e}$ * significativo a $5 \%$ de probabilidade pelo teste $\mathrm{F}$.

Primiano (2006) avaliando a biologia de $D$. saccharalis criadas em dieta artificial contendo colmos de variedades de cana-de-açúcar, concluiu que o período larval da praga variou de 64,40 dias para as lagartas alimentadas com dietas contendo colmo da variedade SP80-3280 e 67,88 dias para as alimentadas com SP89-1115.
Em relação ao período pupal apenas a dieta com $100 \%$ da concentração dos componentes diferiu da dieta com $25 \%$, sendo os valores médios de 8,0 e 9,2 dias respectivamente. Não foram constatadas diferenças entre os tratamentos com a incorporação ou não de partes de colmo das variedades, onde sem diferenciação, os valores médios de 8,7; 8,7 e 9,0 dias foram constatados nos insetos 
alimentados com a variedade SP80-3280, RB835486 e sem incorporação de colmos de variedades, respectivamente (Tabela 1). Esses resultados na presença ou não de partes de colmos de variedades, se apresentaram bastantes próximos àqueles relatados por Bellodi (1993) e Primiano (2006).

Quanto à duração do período total, podese observar na (Tabela 1), que houve diferença significativa entre os tratamentos com 100, 75 e $50 \%$ com valores médios de 35,2; 37,2 e 35,9 dias respectivamente, ao quais proporcionaram melhor desenvolvimento da lagarta, diferindo da dieta com $25 \%$ a qual aumentou a duração deste período $(47,9$ dias). Quanto a presença ou não de partes de colmo das variedades, não foram observadas diferenças, no entanto os dados médios foram de 38,$3 ; 39,5$ e
39,3 dias, respectivamente para a dieta incorporada com a variedade resistente, suscetível e para a não incorporada.

A interação entre as diferentes concentrações de dietas e a presença ou não de colmos de variedades de cana-de-açúcar não diferiram entre si, mostrando que o comparativo entre as intensidades dos componentes nas dietas não foi afetada pela presença ou não dos colmos das variedades (Tabela 1).

A viabilidade larval, pupal e total também diferiram entre si quando as lagartas foram criadas em meios artificiais com concentrações distintas de seus componentes, e não foram afetadas pela presença ou não de partes de colmo das variedades de cana-de-açúcar (Tabela 2).

Tabela 2. Valor médio da viabilidade larval, pupal e total de D. saccharalis, obtidos em quatro dietas de diferentes concentrações de seus componentes, incorporados ou não com partes de colmo de variedades de cana-de-açúcar. Temperatura: $25 \pm 1^{\circ} \mathrm{C}$; UR: $70 \pm 10 \%$; Fotofase: 12 horas.

\begin{tabular}{|c|c|c|c|}
\hline Concentração da dieta (D) ${ }^{1}$ & $\begin{array}{c}\text { Viabilidade larval }^{2} \\
(\%)\end{array}$ & $\begin{array}{c}\text { Viabilidade pupal }{ }^{2} \\
(\%)\end{array}$ & $\begin{array}{c}\text { Viabilidade total }{ }^{2} \\
(\%)\end{array}$ \\
\hline $100 \%$ & $76,01 \mathrm{a}$ & $81,86 \mathrm{a}$ & $70,31 \mathrm{a}$ \\
\hline $75 \%$ & 80,44 a & $78,58 \mathrm{ab}$ & $70,95 \mathrm{a}$ \\
\hline $50 \%$ & 81,14 a & $83,20 \mathrm{a}$ & 75,31 a \\
\hline $25 \%$ & $47,76 \mathrm{~b}$ & $64,69 \mathrm{~b}$ & $37,57 \mathrm{~b}$ \\
\hline F (D) & $21,68^{* *}$ & $4,05^{*}$ & $17,49^{* *}$ \\
\hline D.M.S & 12,76 & 15,77 & 15,61 \\
\hline \multicolumn{4}{|l|}{ Colmo (C) } \\
\hline Com variedade (SP80-3280) & $67,26 \mathrm{a}$ & $77,52 \mathrm{a}$ & $59,22 \mathrm{a}$ \\
\hline Com variedade (RB83-5486) & $76,97 \mathrm{a}$ & $78,91 \mathrm{a}$ & $68,31 \mathrm{a}$ \\
\hline Sem variedade & $69,80 \mathrm{a}$ & $74,81 \mathrm{a}$ & $63,07 \mathrm{a}$ \\
\hline$\overline{F(D)}$ & .. $2,91 \mathrm{NS}$ & $\begin{array}{ll}. . & 0,33^{\mathrm{NS}}\end{array}$ & .. $1,59 \mathrm{NS}$ \\
\hline D.M.S & 10,05 & 12,42 & 12,29 \\
\hline $\mathrm{F}(\mathrm{D} \times \mathrm{C})$ & $0,57^{\mathrm{NS}}$ & $1,17^{\mathrm{NS}}$ & $0,05^{\mathrm{NS}}$ \\
\hline Desvio padrão & 14,47 & 17,90 & 17,70 \\
\hline $\mathrm{CV}(\%)$ & 20,29 & 23,22 & 27,86 \\
\hline
\end{tabular}

${ }^{1}$ Médias seguidas de mesma letra na coluna, para cada fator, não diferem entre si ao nível de $5 \%$ de probabilidade pelo teste de Tukey. ${ }^{\mathrm{NS}}$ não significativo, ${ }^{* *}$ significativo a $1 \%$ e *significativo a $5 \%$ de probabilidade pelo teste $\mathrm{F} .{ }^{2}$ Dados transformados em arc $\operatorname{sen} \sqrt{X / 100}$. 
Analisando a viabilidade larval, nota-se que as dietas com 100, 75 e 50\% da concentração dos componentes diferiram da dieta com $25 \%$, sendo essa última a que apresentou menor índice de viabilidade $(47,76 \%)$, enquanto que as demais apresentaram valores médios maiores de 76,01; 80,44 e 81,14\% respectivamente. Não houve diferença para as lagartas alimentadas ou não com partes de colmo das variedades de cana-de-açúcar, sendo as viabilidades de 67,$26 ; 76,97$ e $69,80 \%$ para as lagartas criadas nas dietas incorporadas com as variedades SP803280, RB83-5486 e aquelas criadas em dietas não incorporadas com partes de colmo de variedades de cana-de-açúcar, respectivamente (Tabela 2).

Para os valores de viabilidade pupal, esses foram afetados pelas concentrações dos componentes das dietas, sendo as dietas com 100 e 50\% da concentração com valores médios de 81,86 e $83,20 \%$ respectivamente, as que proporcionaram maiores viabilidades, diferindo da dieta com $25 \%$ a qual apresentou valor médio de 64,69\%. Embora a viabilidade da fase pupal tenha variado de 77,52; 78,91 e $74,81 \%$ para os indivíduos criados em dieta artificial contendo colmo seco triturado das variedades SP80-3280 e RB83-5486 ou ainda não contendo variedades, respectivamente, não houve diferença estatística entre as médias registradas nesses tratamentos (Tabela 2).

Os valores médios da viabilidade total (Tabela 2) correspondente a 70,31; 70,95 e 75,31\% respectivamente para as diferentes concentrações de dietas de 100, 75 e 50\%, diferiram da dieta com $25 \%$, que obteve um valor médio de $37,57 \%$. Quando comparados na presença ou não de partes de colmos de variedades de cana-de-açúcar, notase que não houve diferenças entre elas, porém os valores médios para as dietas que receberam colmos da variedade SP80-3280, da RB83-5486 e para a que não recebeu parte de colmos das variedades foram respectivamente de 59,22; 68,31 e 63,07\%.

Do mesmo modo que para as análises de período larval, pupal e total, a interação dos fatores (Concentração-dieta $\mathrm{x}$ presença ou não de colmo) para as análises de viabilidade larval, pupal e total não foram significativas (Tabela 3).

Com relação ao peso larval e pupal, observaramse diferenças entre os insetos quando comparados quanto às diferentes concentrações dos componentes das respectivas dietas. $\mathrm{O}$ mesmo ocorreu quando comparados na presença ou não de partes de colmos de plantas de cana-de-açúcar (Tabela 3).

Quanto ao peso larval, observa-se que houve diferença entre todos os tratamentos, sendo que a dieta com concentração $100 \%$ foi a que proporcionou maior ganho de peso para as larvas $(0,0549 \mathrm{~g})$, quando comparada com as demais. Já a dieta com $25 \%$ da concentração foi a que apresentou larvas menos pesadas $(0,0142 \mathrm{~g})$, sugerindo ser esta a menos adequada (Tabela 3 ).

Também houve diferenças entre as lagartas com incorporação de colmo da variedade SP803280 nas dietas, as quais apresentaram peso médio de $0,0440 \mathrm{~g}$, diferindo do peso médio das lagartas criadas nas dietas com partes de colmo da variedade RB83-5486, e na dieta sem incorporação de variedades, as quais apresentaram pesos médios menores de 0,0324 e $0,0381 \mathrm{~g}$, respectivamente. Este fato evidencia que esses dois últimos materiais mostram-se menos adequados ao desenvolvimento do inseto, semelhante aos relatos por Bellodi (1993) quanto à utilização da variedade resistente.

Quanto ao peso pupal, nota-se que as dietas com 100,75 e $50 \%$ da concentração dos componentes provocaram um aumento nos valores médios deste parâmetro, sendo de 0,$1539 ; 0,1458$ e $0,1375 \mathrm{~g}$, respectivamente, quando comparados com o meio artificial com $25 \%$ da concentração dos componentes da dieta padrão, o qual apresentou um valor médio de $0,1073 \mathrm{~g}$. Quanto a presença ou não de colmos de variedades de cana-de-açúcar, apenas o tratamento onde os insetos de $D$. saccharalis foram criados nas dietas sem incorporação de partes de colmo de variedades, diferiu da dieta que foi incorporada com a variedade resistente (RB83-5486), que obteve um peso pupal médio de $0,1245 \mathrm{~g}$, evidenciando ser menos adequada ao desenvolvimento do inseto (Tabela 3). 
Tabela 3. Valor médio do peso larval, pupal e da longevidade do adulto de $D$. saccharalis, obtidos em quatro dietas de diferentes concentrações de seus componentes, incorporados ou não com partes de colmo de variedades de canade-açúcar. Temperatura: $25 \pm 1^{\circ} \mathrm{C}$; UR: $70 \pm 10 \%$; Fotofase: 12 horas.

\begin{tabular}{lcll}
\hline \multicolumn{1}{c}{ Concentração da dieta $(\mathrm{D})^{1}$} & \multicolumn{1}{c}{$\begin{array}{c}\text { Peso larval } \\
(\mathrm{mg})\end{array}$} & $\begin{array}{c}\text { Peso pupal } \\
\text { (gramas) }\end{array}$ & $\begin{array}{c}\text { Longevidade do adulto } \\
\text { (dias) }\end{array}$ \\
\hline $100 \%$ & $54,90 \mathrm{a}$ & $153,90 \mathrm{a}$ & $3,70 \mathrm{~b}$ \\
$75 \%$ & $36,20 \mathrm{c}$ & $145,80 \mathrm{a}$ & $4,80 \mathrm{a}$ \\
$50 \%$ & $47,50 \mathrm{~b}$ & $137,50 \mathrm{a}$ & $4,70 \mathrm{a}$ \\
$25 \%$ & $14,20 \mathrm{~d}$ & $107,30 \mathrm{~b}$ & $5,20 \mathrm{a}$ \\
\hline F ( D ) & $81,76^{* *}$ & $\ldots .8,90^{* *}$ & $6,40^{* *}$ \\
D.M.S & 0,0073 & $\ldots .0,0255$ & 1,00 \\
\hline \multicolumn{1}{c}{ Colmo ( C) } & & \\
\hline Com variedade (SP80-3280) & $44,00 \mathrm{a}$ & $136,30 \mathrm{ab}$ & $4,40 \mathrm{a}$ \\
Com variedade (RB83-5486) & $32,40 \mathrm{~b}$ & $124,50 \mathrm{~b}$ & $4,90 \mathrm{a}$ \\
Sem variedade & $38,10 \mathrm{~b}$ & $147,60 \mathrm{a}$ & $4,50 \mathrm{a}$ \\
\hline F (D) & $11,57^{* *}$ & $3,82^{*}$ & $1,30 \mathrm{NS}$ \\
D.M.S & 0,0058 & 0,0201 & 0,80 \\
\hline F (D x C) & $8,24^{* *}$ & $1,0746^{\mathrm{NS}}$ & $0,60^{\mathrm{NS}}$ \\
\hline Desvio padrão & 0,0083 & 0,0289 & 1,10 \\
CV(\%) & 21,81 & 21,25 & 23,60 \\
\hline
\end{tabular}

${ }^{1}$ Médias seguidas de mesma letra na coluna, para cada fator, não diferem entre si ao nível de $5 \%$ de probabilidade pelo teste de Tukey. ${ }^{\mathrm{NS}}$ não significativo, ${ }^{* *}$ significativo a $1 \%$ e ${ }^{*}$ significativo a $5 \%$ de probabilidade pelo teste $\mathrm{F}$.

A longevidade dos adultos diferiu entre si quando avaliada sob diferentes concentrações de dietas, sendo que as dietas com 25,50 e $75 \%$ da concentração dos componentes da dieta padrão apresentaram adultos mais longevos com de 5,2; 4,7 e 4,8 dias respectivamente, quando comparados aos adultos provenientes de larvas criadas na dieta com $100 \%$ (3,7 dias). Não houve diferença significativa quanto à presença ou não de partes de colmos de variedades de cana-de-açúcar quanto à longevidade dos adultos, sendo os valores médios de 4,4 dias para os insetos criados na dieta com a incorporação da variedade SP80-3280, de 4,9 dias para os alimentados com a dieta incorporada com a variedade RB83-5486 e de 4,5 dias para os criados em dietas não incorporadas com partes de colmos de cana-de-açúcar (Tabela 3).

Os valores observados para o parâmetro, diferentes concentrações dos componentes nas dietas, foram semelhantes aos de Walker et al. (1996) citado por Adkisson et al. (1960), onde apresentaram a dieta com $100 \%$ da concentração dos componentes de uma dieta padrão foi a mais eficiente para o desenvolvimento do inseto.

Com relação ao peso larval, pupal e longevidade do adulto, a interação dos fatores (Concentração da dieta $\mathrm{x}$ presença ou não de colmo) foi significativa apenas na avaliação do peso larval (Tabela 3 ).

Pela análise desta interação (Tabela 4), ao observar o efeito da concentração dos componentes das diferentes dietas dentro da presença ou não de colmos de variedades de cana-de-açúcar, apenas as concentrações 100 e $50 \%$ obtiveram diferenças, sendo que no caso da concentração $100 \%$ os valores que diferiram entre si foram das dietas incorporadas com a variedade SP80-3280 com as dietas não incorporadas com colmos de cana-de-açúcar, com valores médios de $0,0653 \mathrm{~g}$ e $0,0450 \mathrm{~g}$, respectivamente. Já na dieta com 50\%, com incorporação da variedade suscetível (SP803280) e sem incorporação, com valores médios de 0,0615 e $0,0524 \mathrm{~g}$ respectivamente, diferiram das incorporadas com a variedade resistente (RB835486) com um índice médio inferior de $0,0286 \mathrm{~g}$ (Tabela 4). 
Tabela 4. Valor médio transformado da análise de desdobramento referente à interação entre dietas de diferentes concentrações de seus componentes versus a presença ou não de colmos de cana-de-açúcar, para o peso larval (gramas) de D. saccharalis. Temperatura: $25 \pm 1^{\circ} \mathrm{C}$; UR: $70 \pm 10 \%$; Fotofase: 12 horas.

\begin{tabular}{lllll}
\hline \multirow{2}{*}{ Concentração } & \multicolumn{3}{c}{ Presença ou não de colmo $^{1}$} & \multirow{2}{*}{ Teste F (C) } \\
\cline { 2 - 4 } & Variedade SP80-3280 & Variedade RB83-5486 & Sem variedade & \\
\hline $100 \%$ & $0,0653 \mathrm{Aa}$ & $0,0543 \mathrm{ABa}$ & $0,0450 \mathrm{Bab}$ & $8,98^{* *}$ \\
$75 \%$ & $0,0360 \mathrm{Ab}$ & $0,0373 \mathrm{Ab}$ & $0,0353 \mathrm{Ab}$ & $0,09^{\mathrm{NS}}$ \\
$50 \%$ & $0,0615 \mathrm{Aa}$ & $0,0286 \mathrm{Bb}$ & $0,0524 \mathrm{Aa}$ & $24,89^{* *}$ \\
$25 \%$ & $0,0133 \mathrm{Ac}$ & $0,0095 \mathrm{Ac}$ & $0,0198 \mathrm{Ac}$ & $2,33^{\mathrm{Ns}}$ \\
\hline Teste F (D) & $51,79^{* *}$ & $30,10^{* *}$ & $17,55^{* *}$ & - \\
\hline
\end{tabular}

${ }^{1}$ Médias seguidas de mesma letra maiúscula na linha, e minúscula na coluna, para cada fator, não diferem entre si ao nível de 5\% de probabilidade pelo teste de Tukey. ${ }^{\text {NS }}$ não significativo, ${ }^{* *}$ significativo a $1 \%$ de probabilidade pelo teste $\mathrm{F}$.

Nota-se que para essa última concentração de dieta, a redução do peso médio das lagartas alimentadas com a variedade suscetível para as alimentadas com a variedade resistente foi de aproximadamente $54 \%$, possibilitando com isso uma melhor discriminação entre os genótipos resistente e suscetível.

Quanto ao efeito da presença ou não de colmos de variedades de cana-de-açúcar dentro das concentrações das dietas (Tabela 4), todas as dietas incorporadas ou não com colmos apresentaram diferenças, sendo que no geral a dieta com $25 \%$ da concentração dos componentes da dieta padrão foi a que apresentou os menores índices médios para o peso das lagartas, afetando o desenvolvimento da D. saccharalis.

\section{Conclusões}

A dieta com 100\% da concentração de seus componentes é mais favorável ao desenvolvimento de D. saccharalis, enquanto aquela com $25 \%$ é menos favorável.

A dieta com $100 \%$ da concentração de seus componentes associada à presença de colmo da variedade SP80-3280 (suscetível) proporciona maior peso larval.

Os pesos larval e pupal do inseto são afetados negativamente quando incorpora-se na dieta colmos secos triturados da variedade RB83-5486 (resistente).
A dieta com $50 \%$ da concentração de seus componentes proporciona, quanto ao peso larval, melhor discriminação entre os genótipos resistente e suscetível à broca da cana-de-açúcar.

\section{Referências}

ADKISSON, P. L.; VANDERZANT, E. S.; BULL, D. L.; ALLSON, W. E. A wheat germ medium for rearing the pink bolloworm. Journal of Economic Entomology, Lanham, v. 41, p. 651-653, 1960.

AGRIANUAL: Anuário da agricultura brasileira. São Paulo: FNP Consultoria e Comércio, 2009. p. 235-259.

BELLODI, M. P. Influência de colmos de variedades de cana-de-açúcar, incorporados em dieta artificial, no desenvolvimento de Diatraea saccahralis (Fabr.) e no parasitismo de Cotesia flavipes (Cam.). 1993. Trabalho de Conclusão de Curso (Graduação em Agronomia) - Faculdade de Ciências Agrárias e Veterinárias. Universidade Estadual Paulista, Jaboticabal.

BESSIN, R. T.; MOSER, E. B.; REAGAN, T. E. Integration of control: tactics for management of the sugarcane borer (Lepdoptera: Pyralidae) in Louisiana sugarcane. Journal of Economic Entomology, Lanham, v. 83, n. 4, p. 1563-1569, 1990.

BOIÇA JUNIOR, A. L.; LARA, F. M.; BELLODI, M. P. Influência de variedades de cana-de-açúcar, incorporadas em dieta artificial, no desenvolvimento de Diatraea saccharalis (Fabr.) e no seu parasitismo por Cotesia flavipes (Cam.). Anais da Sociedade Entomológica do Brasil, Piracicaba, v. 26, n. 3, p. 537-550, 1997.

DERNEIKA, O. Resistência da cana-de-açúcar a Diatraea saccharalis (Fabr., 1794) (LepidópteraPyralidae): Comportamento de variedades em três cortes e em quatro locais do Estado de São Paulo.1985. 
Dissertação (Mestrado em Agronomia) - Programa de Pós-graduação em Entomologia Agrícola. Faculdade de Ciências Agrárias e Veterinárias. Universidade Estadual Paulista, Jaboticabal.

GALLO, D. Infestação da broca nas principais variedades de cana. Revista de Agricultura, Piracicaba, v. 29, n. 5, p. 149-155, 1954.

GALLO, D.; NAKANO, O.; SILVEIRA NETO, S.; BAPTISTA, G. C.; BERTI FILHO, E.; PARRA, J. R. P.; ZUCCHI, R. A.; ALVES, S. B.; VENDRAMIM, J. D.; MARCHINI, L. C.; LOPES, J. R. S.; OMOTO, S. Entomologia agrícola. Piracicaba: FEALQ, 2002. 920 p.

HENSLEY, S. D.; HAMMOND, A. M. Laboratory techniques for rearing the sugar cane borer on an artificial diet. Journal Economic Entomology, Lanham, v. 61, p. 1742-1743, 1968.

LARA, F. M. Princípios de resistência de plantas a insetos. 2. ed. São Paulo: Ícone, 1991. 336 p.

MARCONATO, J. R. Aspectos biológicos de Diatraea saccharalis (Fabr.; 1794) (Lepidoptera- Pyralidae) em meio artificial contendo diferentes genótipos de sorgo e milho na forma de colmos secos e triturados. 1988. Dissertação (Mestrado em Agronomia) - Programa de Pós-Graduação em Entomologia Agrícola. Faculdade de Ciências Agrárias e Veterinárias. Universidade Estadual Paulista, Jaboticabal.

PRIMIANO, G. S. Avaliação da resistência de variedades de cana-de-açúcar (Saccharum officinarum L.) a Diatraea saccharalis (Fabr.; 1794) (Lep.Crambidae). 2006. Trabalho de Conclusão de Curso (Graduação em Agronomia) - Faculdade de Ciências Agrárias e Veterinárias. Universidade Estadual Paulista, Jaboticabal.

TERÁN, F. O.; PRECETTI, A. A. C. M.; DERNEIKA, O. Broca da cana-de-açúcar: diatraea saccharalis. Piracicaba: Copersucar, 1983. 15 p.

TERÁN, F. O.; SÁNCHEZ, A. G.; PRECETTI, A. A. C. M. Importância econômica, aspectos bioecológicos e manejo populacional da broca da cana-deaçúcar, Diatraea saccharalis. In: SEMINÁRIO DE TECNOLOGIA AGRONÔMICA, 2., 1986, Piracicaba. Anais... Piracicaba: Copersucar, 1986. p. 153-167. 